\title{
Associação do sobrepeso com variáveis sócio-demográficas e estilo de vida em universitários
}

\author{
Association of overweight with socio-demographic variables \\ and lifestyle among Brazilian university students
}

Diego Augusto Santos Silva ${ }^{1}$

Teresa Maria Bianchini de Quadros ${ }^{2}$

Alex Pinheiro Gordia ${ }^{2}$

Edio Luiz Petroski ${ }^{1}$

\footnotetext{
${ }^{1}$ Grupo de Pesquisa em Cineantropometria e Desempenho Humano, Programa de PósGraduação em Educação Física, Centro de Desportos, Universidade Federal de Santa Catarina. Campus Universitário Reitor João David Ferreira Lima, Trindade. 88040-970 Florianópolis SC.

diegoaugustoss@yahoo.com.br ${ }^{2}$ Centro de Formação de Professores Amargosa, Universidade do Recôncavo da Bahia
}

\begin{abstract}
Objective: To investigate the association of overweight with socio-demographic variables and lifestyle among freshmen of a Brazilian public university. Participants: A total of 685 students were evaluated in April 2008. Methods: Overweight was determined based on body mass index. Lifestyle and socio-demographic variables were established using a self-administered questionnaire. Results: Overweight was observed in $16 \%$ of the students and lifestyle was inadequate in $5.1 \%$. The incidence of overweight was higher among male students (OR=2.69; 95\%CI: 1.64$4.42)$, students aged $\geq 20$ years $(O R=2.01 ; 95 \% C I$ : 1.25-3.25), married students ( $O R=2.44 ; 95 \% C I$ : 1.11-5.40), and students who reported being more than $2 \mathrm{~kg}$ above healthy weight $(\mathrm{OR}=17.05$; 95\%CI: 7.73-37.63) and ingesting caffeine-containing beverages more than twice a day $(\mathrm{OR}=$ 1.66; 95\%CI: 1.00-2.75). Conclusion: This study demonstrates the need for guidance of university students regarding a healthy lifestyle and for physical activity programs on the campus aimed at reducing excess weight, with special attention to male, older and married students.
\end{abstract}

Key words Body Mass Index, Lifestyle, Overweight, Socio-demographic variables, Overweight
Resumo Objetivo: Analisar a associação do sobrepeso com variáveis sociodemográficas e estilo de vida de calouros de uma universidade pública brasileira. Participantes: 685 calouros avaliados em Abril de 2008. Métodos: O sobrepeso foi verificado pelo indice de massa corporal. O estilo de vida $e$ as variáveis sociodemográficas foram estabelecidos através de um questionário auto administrado. Resultados: apresentaram sobrepeso $16 \%$ dos calouros e 5,1\% estilo de vida inadequado. Universitários do sexo masculino $(O R=2,69$; 95\%IC: 1,64-4,42), com idade $\geq 20$ anos ( $O R=2,01$; 95\%IC: 1,25-3,25), casados (OR=2,44; 95\%IC: 1,11-5,40), que reportaram estar mais de $2 \mathrm{~kg}$ acima do peso saudável $(O R=17,05 ; 95 \% I C: 7,73-37,63)$ e que reportaram ingerir bebidas com cafeina mais de duas vezes por dia (OR=1,66; 95\%IC: 1,00-2,75), tiveram mais chance de sobrepeso. Conclusão: $O$ estudo evidencia a necessidade de orientação do universitário para um estilo de vida saudável e da oferta de programas de atividades físicas no campus que vise à redução do excesso de peso, com atenção especial aos universitários do sexo masculino, mais velhos e casados.

Palavras-chave Índice de massa corporal, Estudantes universitários, Estilo de vida, Variáveis sócio-demográficas, Sobrepeso 


\section{Introdução}

A prevalência de sobrepeso vem aumentando durante as últimas décadas em populações de diferentes países, afetando todas as idades, etnias, sexo, diferentes condições econômicas e nível de escolaridade, preocupando setores públicos devido à associação com vários agravos à saúde $\mathrm{e}^{1-4}$.

$\mathrm{O}$ ingresso no ensino superior é considerado um momento de transição na vida das pessoas ${ }^{5}$, pois ocorrem mudanças no estilo de vida, resultantes do aumento de responsabilidades e de afazeres $^{6,7}$. Ademais, pesquisas já reportaram que ao ingressar na universidade há um aumento do nível de estresse ${ }^{6,8}$, alteração dos hábitos alimen$\operatorname{tares}^{9} \mathrm{e}$ redução da atividade física ${ }^{7}$, o que reflete no incremento do sobrepeso neste segmento populacional $^{10,11}$.

A atividade física e os hábitos alimentares são dois elementos do estilo de vida que desempenham um papel significativo na promoção da saúde e na prevenção de diversas doenças ${ }^{12}$. Aliado a isso, outros elementos do estilo de vida são também significativos para a saúde e o bem-estar, tais como evitar o uso de cigarros e o consumo de álcool, possuir um bom relacionamento com a família e amigos, prática de sexo seguro, além da necessidade de possuir uma visão otimista e positiva da vida ${ }^{13}$.

O conhecimento das variáveis sociodemográficas, como o sexo, a faixa etária, o estado civil, o nível socioeconômico e o turno de estudo e trabalho, auxiliam na identificação daqueles subgrupos mais expostos a doenças como a obesidade ${ }^{4,5,14}$.

Neste sentido, a compreensão dos fatores associados ao sobrepeso em universitários pode ajudar em campanhas e na oferta de atividades mais efetivas e variadas, para os acadêmicos com excesso de peso corporal com estilo de vida inadequado. Assim, o objetivo do presente estudo foi analisar a associação do sobrepeso com variáveis sócio-demográficas e indicadores do estilo de vida de calouros de uma universidade pública brasileira.

\section{Métodos}

\section{População e Amostra}

Este estudo foi realizado com base no banco de dados do projeto de pesquisa "Avaliação da aptidão física relacionada à saúde de universitários da Universidade Federal de Santa Catarina (UFSC)". Os protocolos de intervenção no estu- do foram aprovados pelo Comitê de Ética em Pesquisa com seres humanos da UFSC.

A população de estudo foi composta por 2290 universitários, ingressantes na universidade no primeiro semestre de 2008. Para o cálculo do tamanho da amostra, foram seguidos os procedimentos de Luiz e Magnanini ${ }^{15}$, adotando-se um nível de confiança de $95 \%$, prevalência estimada de $30 \%$ e um erro máximo permitido de 3,0 pontos percentuais. Após os cálculos, estimou-se que seria necessário avaliar 644 universitários.

Utilizou-se a amostragem estratificada proporcional por centro de ensino e turno de estudo (diurno e noturno). Dentro de cada centro de ensino, utilizou-se o procedimento conglomerado de turmas, participando da coleta de dados todos os alunos presentes em sala de aula no dia da avaliação. Como não houve recusa em assinar o Termo de Consentimento Livre e Esclarecido e responder os questionários, a amostra final foi formada por 685 calouros ( 410 do sexo masculino e 275 do sexo feminino), com média de $20,4(\mathrm{dp}=3,1)$ anos de idade.

\section{Instrumentos e Procedimentos}

A massa corporal $(\mathrm{kg})$ e a estatura $(\mathrm{cm})$ foram utilizadas para o cálculo do IMC $(\mathrm{kg} / \mathrm{m} 2)$, sendo estas obtidas através de autorelato, procedimento validado para adultos brasileiros ${ }^{16} \mathrm{e}$ utilizado extensivamente na população universitária $^{10}$. Para a classificação do sobrepeso, utilizaram-se os pontos de corte estabelecidos pela Organização Mundial da Saúde ${ }^{17}$ (IMC e" $25 \mathrm{~kg} / \mathrm{m} 2$ ).

Para a determinação do estilo de vida, foi utilizado o questionário auto-administrado "Estilo de Vida Fantástico", da Associação Canadense de Fisiologia ${ }^{12}$, traduzido para o português e validado para a população brasileira por Rodriguez-Añez et al. ${ }^{18}$. De acordo com os critérios de classificação deste instrumento ${ }^{13,18}$, o estilo de vida é representado por cinco categorias. No presente estudo, foram considerados como estilo de vida "adequado" os sujeitos das categorias "Excelente", "Muito bom" e "Bom", e estilo de vida "inadequado" os da "Regular" e "Necessita melhorar".

$\mathrm{O}$ instrumento possui 25 indicadores divididos em nove domínios: 1) família e amigos; 2) atividade física; 3 ) nutrição; 4) tabaco e tóxicos; 5) álcool; 6) sono, cinto de segurança, estresse e sexo seguro; 7) tipo de comportamento; 8) introspecção; 9) trabalho. As respostas dos universitários a cada comportamento foram dicotomizadas em condição "adequada" ou "inadequada" para saúde. 
As variáveis sócio-demográficas analisadas foram: sexo (masculino e feminino), faixa etária $(<20$ e $\geq 20$ anos), estado civil (solteiro e casado), trabalho (sim e não), turno de estudo (diurno e noturno) e escolaridade da mãe e do pai ( $\leq$ 4 anos de estudo, 5-8 anos e $\geq 9$ anos).

\section{Tratamento estatístico}

Para analisar diferenças percentuais entre os indicadores do estilo de vida e variáveis sociodemográficas para o estado nutricional dos universitários, foi empregado o teste qui-quadrado. A regressão logística binária (bruta e ajustada) foi usada para examinar as associações entre o estado nutricional (variável dependente) com as condições do estilo de vida e aspectos sócio-demográficos (variáveis independentes). Todas as variáveis do estilo de vida e sócio-demográficas investigadas, cujas associações com o estado nutricional apresentaram valores de $\mathrm{p}<0,25$ no teste qui-quadrado, foram introduzidas no modelo de regressão, uma a uma, de maneira crescente, conforme sua significância estatística e/ou que foram consideradas relevantes de acordo com a literatura. O nível de significância foi estabelecido em $5 \%$.

\section{Resultados}

A prevalência de sobrepeso nos universitários investigados foi de $16 \%$. A média do IMC foi $22,12(\mathrm{dp}=3,09) \mathrm{kg} / \mathrm{m}^{2}$. Ao considerar a análise do questionário do estilo de vida, $5,1 \%$ dos calouros foram classificados com estilo de vida inadequado (dados não apresentados).

Os resultados referentes à associação entre o estado nutricional e as variáveis sócio-demográficas estão descritas na Tabela 1. Na análise bruta, as variáveis determinantes do sobrepeso foram: o sexo, a faixa-etária, o estado civil, o trabalho, o turno de estudo e a escolaridade da mãe. Os resultados na análise ajustada indicaram que os universitários do sexo masculino ( $\mathrm{OR}=2,69$; 95\% IC: $1,64-4,42)$, casados (OR $=2,44 ; 95 \%$ IC: $1,11-5,40)$, e com 20 anos ou mais de idade (OR=2,01; 95\%IC: $1,25-3,25)$ foram os calouros com maiores chances de apresentar sobrepeso.

Os resultados referentes à associação entre o estado nutricional e os indicadores do estilo de vida podem ser observados na tabela 2. De acordo com a análise bruta, o risco para sobrepeso foi maior entre universitários que relataram estar com mais de dois quilos acima do peso considerado saudável, os que se sentem tensos e desapontados, os que não possuem uma dieta ba-

Tabela 1. Análise de regressão logística entre o sobrepeso e variáveis sociodemográficas de universitários brasileiros

\begin{tabular}{lccc}
\hline \multicolumn{1}{c}{ Variáveis Sociodemográficas } & $\begin{array}{c}\text { Excesso de Peso } \\
(\%)\end{array}$ & $\begin{array}{c}\text { Análise Bruta } \\
\text { OR (IC 95\%) }\end{array}$ & $\begin{array}{c}\text { Análise Ajustada } \\
\text { OR (IC 95\%) }\end{array}$ \\
\hline Sexo & 9,5 & $\mathrm{p}<0,001$ & $\mathrm{p}<0,001$ \\
$\quad$ Feminino & 20,2 & 1 & 1 \\
$\quad$ Masculino & & $2,43(1,52-3,89)$ & $2,69(1,64-4,42)$ \\
Faixa-etária & 11,3 & $\mathrm{p}<0,001$ & $\mathrm{p}=0,004$ \\
$\quad<20$ anos & 23,9 & $2,47(1,62-3,74)$ & 1 \\
$\quad 20$ anos & & $\mathrm{p}=0,003$ & $2,01(1,25-3,25)$ \\
Estado Civil & 14,9 & 1 & $\mathrm{p}=0,027$ \\
$\quad$ Solteiro & 33,3 & $2,86(1,42-5,77)$ & 1 \\
$\quad$ Casado & & $\mathrm{p}=0,005$ & $2,44(1,11-5,40)$ \\
Trabalho & 13,7 & 1 & $\mathrm{p}=0,635$ \\
$\quad$ Não & 23 & $1,89(1,22-2,94)$ & 1 \\
$\quad$ Sim & & $\mathrm{p}=0,008$ & $1,13(0,68-1,90)$ \\
Turno de Estudo & 13,6 & 1 & $\mathrm{p}=0,207$ \\
$\quad$ Diurno & 21,9 & $1,78(1,16-2,73)$ & 1 \\
$\quad$ Noturno & & $\mathrm{p}=0,027$ & $1,35(0,85-2,15)$ \\
Escolaridade Materna & 14,4 & 1 & $\mathrm{p}=0,379$ \\
$\geq 9$ anos & 19,4 & $1,44(0,76-2,70)$ & 1 \\
5-8 anos & 28,6 & $2,38(1,23-4,63)$ & $1,63(0,80-3,36)$ \\
$\quad \leq 4$ anos & & & $0,95(0,48-1,89)$ \\
\hline
\end{tabular}

OR: odds ratio; IC: intervalo de confiança. 
Tabela 2. Análise de regressão logística entre o excesso de peso corporal e indicadores do estilo de vida de universitários brasileiros.

\begin{tabular}{|c|c|c|c|}
\hline Indicadores do Estilo de Vida & $\begin{array}{l}\text { Excesso de } \\
\text { Peso }(\%)\end{array}$ & $\begin{array}{l}\text { Análise Bruta OR } \\
\text { (IC 95\%) }\end{array}$ & $\begin{array}{l}\text { Análise Ajustada } \\
\text { OR (IC 95\%) }\end{array}$ \\
\hline Estar no intervalo de quantos quilos do peso saudável & & $\mathrm{p}<0,001$ & $\mathrm{p}<0,001$ \\
\hline $0-2 \mathrm{~kg}$ & 2,2 & 1 & 1 \\
\hline Mais que $2 \mathrm{~kg}$ & 27,8 & $17,10(7,81-37,42)$ & $17,05(7,73-37,63)$ \\
\hline Sentir-se tenso e desapontado & & $\mathrm{p}=0,017$ & $\mathrm{p}=0,203$ \\
\hline Não & 12,4 & 1 & 1 \\
\hline Sim & 19,2 & $1,67(1,10-2,54)$ & $1,36(0,85-2,20)$ \\
\hline Dieta balanceada & & $\mathrm{p}=0,019$ & $\mathrm{p}=0,198$ \\
\hline Sim & 11,9 & 1 & 1 \\
\hline Não & 18,6 & $1,69(1,09-2,63)$ & $1,38(0,85-2,25)$ \\
\hline Ingerir bebidas com cafeína (café, chá ou coca-cola) & & $\mathrm{p}=0,030$ & $\mathrm{p}=0,050$ \\
\hline $1-2$ vezes por dia & 14,2 & 1 & 1 \\
\hline Mais que 2 vezes por dia & 21,3 & $1,64(1,05-2,56)$ & $1,66(1,00-2,75)$ \\
\hline Ingestão média de álcool por semana & & $\mathrm{p}=0,051$ & $\mathrm{p}=0,083$ \\
\hline$\leq 7$ doses & 14,3 & 1 & 1 \\
\hline$>7$ doses & 20,7 & $1,56(1,00-2,44)$ & $1,56(0,94-2,57)$ \\
\hline Ter alguém para conversar as coisas importantes & & $\mathrm{p}=0,054$ & $\mathrm{p}=0,113$ \\
\hline Sim & 14,5 & 1 & 1 \\
\hline Não & 21,1 & $1,58(1,00-2,50)$ & $1,73(0,88-3,39)$ \\
\hline Dar e receber afeto & & $\mathrm{p}=0,699$ & $\mathrm{p}=0,088$ \\
\hline Sim & 15,6 & 1 & 1 \\
\hline Não & 16,9 & $0,91(0,57-1,46)$ & $1,80(0,92-3,54)$ \\
\hline $\begin{array}{l}\text { Exagerar freqüentemente no açúcar, sal, gordura } \\
\text { animal, salgadinhos. }\end{array}$ & & $\mathrm{p}=0,094$ & $\mathrm{p}=0,674$ \\
\hline Nenhum item & 16,9 & 1 & 1 \\
\hline Um ou mais itens & 10,4 & $1,76(0,90-3,41)$ & $1,17(0,56-2,44)$ \\
\hline Dormir bem & & $\mathrm{p}=0,127$ & $\mathrm{p}=0,470$ \\
\hline Sim & 14 & 1 & 1 \\
\hline Não & 18,3 & $1,38(0,91-2,07)$ & $1,18(0,75-1,87)$ \\
\hline Ser moderadamente ativo & & $\mathrm{p}=0,819$ & $\mathrm{p}=0,916$ \\
\hline$\geq 3$ vezes por semana & 16,2 & 1 & 1 \\
\hline$<3$ vezes por semana & 15,5 & $0,95(0,62-1,44)$ & $0,98(0,61-1,55)$ \\
\hline
\end{tabular}

OR: odds ratio; IC: intervalo de confiança.

lanceada, que ingerem bebidas com cafeína mais de duas vezes por dia, e com uma média de ingestão de álcool por semana superior a sete doses. Os resultados da análise ajustada indicaram que a chance para apresentar sobrepeso permaneceu significativamente maior entre universitários que reportaram estar com mais de dois quilos acima do peso considerado saudável ( $O R=17,05 ; 95 \%$ IC: 7,73-37,63) e que relataram ingerir bebidas com cafeína mais de duas vezes por dia $(\mathrm{OR}=$ 1,66; 95\% IC: 1,00-2,75).

\section{Discussão}

Dos acadêmicos brasileiros investigados, 16\% apresentaram sobrepeso corporal. Prevalência muito próxima (15\%) foi reportada em estudo realizado com calouros de uma universidade norte-americana ${ }^{9}$. Contudo, a prevalência do presente estudo foi inferior a encontrada em calouros americanos de uma instituição rural ${ }^{18}$ e a observada em pesquisas com não calouros ${ }^{5,10}$. No entanto, considerando que há uma tendência de incremento do peso corporal e do sobrepeso com o avançar dos anos acadêmicos ${ }^{9}$, a prevalência encontrada no presente estudo chama atenção, principalmente, por se tratar de pessoas jovens.

No presente estudo, foi encontrado que 5,1\% dos calouros apresentaram estilo de vida inadequado. Kamien e Power ${ }^{19}$, utilizando o mesmo questionário de estilo de vida, encontraram que 23\% dos acadêmicos australianos apresentaram inadequação nos comportamentos do estilo de 
vida. A presente investigação é a primeira a utilizar o questionário "Estilo de vida Fantástico" em universitários brasileiros, por isso a comparação dos resultados com dados brasileiros fica limitada.

Entre os calouros investigados foi identificado que indivíduos do sexo masculino apresentaram 2,69 vezes mais chances de ter sobrepeso do que seus pares do sexo feminino. Razões de chances próximas a do presente estudo foram reportados em outros países ${ }^{5,20}$, inclusive com calou$\operatorname{ros}^{8}$. Contudo, as investigações que analisaram alterações no peso corporal e na prevalência de sobrepeso durante os anos acadêmicos, não chegaram às mesmas conclusões ${ }^{8,21}$. Holm-Denoma et al. ${ }^{21}$, em um estudo prospectivo, identificaram que universitários do sexo masculino apresentaram uma prevalência mais elevada de sobrepeso do que o feminino durante todo o primeiro ano de faculdade. Em contrapartida, Economos et al. ${ }^{8}$ encontraram que, ao final da faculdade, as mulheres apresentavam uma maior prevalência de sobrepeso do que os homens. Em relação a universitários brasileiros, fazem-se necessários estudos prospectivos para verificar como esta variável se comporta durante o curso universitário.

Os calouros brasileiros avaliados, com 20 anos de idade ou mais, apresentaram, aproximadamente, duas vezes mais chance de ter sobrepeso do que seus pares mais jovens. HajianTilaki e Heidari ${ }^{22}$ verificaram que o padrão do excesso de peso e obesidade abdominal aumenta com a idade, tornando-se um risco à saúde. Ademais, a idade tem sido considerada como um fator prognóstico para o sobrepeso em algumas pesquisas $^{23}$.

Acadêmicos casados apresentaram maior risco para possuir sobrepeso em relação aos solteiros. Estes resultados corroboram dados de diferentes países ${ }^{4,24}$ que encontraram o dobro de chance de pessoas casadas possuírem excesso de peso em comparação aos solteiros. Este achado pode estar relacionado a mudanças no estilo de vida após o casamento, tais como a diminuição da atividade física ${ }^{24}$ e mudança da dieta padrão ${ }^{25}$.

Estudos relatam que a população universitária é capaz de perceber quando apresenta alterações no peso corpóreo ${ }^{26}$. No presente estudo, os calouros que relataram estar no intervalo de mais de dois quilos do peso considerado saudável apresentaram 17,05 vezes mais chance de serem diagnosticados com sobrepeso, constatando que os universitários brasileiros perceberam estas alterações.

Foi reportado na presente pesquisa que a ingestão de bebidas cafeinadas (café, chá ou refrige- rante), duas ou mais vezes por dia, representou 1,65 vezes mais chance de sobrepeso. A inter-relação cafeína, excesso de peso e saúde não está bem elucidada na literatura, pois a combinação de cafeína com outras substâncias, como a epinefrina e o chá verde pode ajudar na redução do peso corporal $^{27}$, já a combinação de cafeína e sacarose pode ter efeito contrário ${ }^{28}$. De acordo com a literatura, a ingestão de refrigerantes cafeinados é um dos preditores da síndrome metabólica ${ }^{29}$, e o consumo de outras bebidas com cafeína pode prejudicar as horas de sono ${ }^{30}$, que por sua vez mostrou-se associado com o ganho de peso e de obesidade $^{30}$. Desta maneira, recomenda-se uma atenção maior ao ingerir bebidas cafeinadas, pois a associação de algumas substâncias pode representar um fator de risco para o sobrepeso.

Era esperada uma associação entre o excesso de peso e outros indicadores do estilo de vida, como por exemplo, atividade física ${ }^{31}$ e dieta balanceada ${ }^{32}$, que juntos podem ser úteis no combate ao sobrepeso. No entanto, o presente estudo teve um "design" transversal que não permitiu identificar relações de causalidade e temporalidade entre o sobrepeso e as outras variáveis, o que pode ter limitado o poder de associação. Outra limitação do presente estudo foi o fato da investigação ter ocorrido em uma instituição pública de ensino superior, cujos dados não podem ser extrapolados para universidades particulares do Brasil.

Tendo em vista a escassez de estudos que verificaram a prevalência de excesso de peso corporal e sua relação com variáveis sociodemográficas e do estilo de vida com a população universitária no Brasil, sobretudo com calouros, o presente estudo auxiliou para o avanço do conhecimento sobre a relação entre estas variáveis extensivamente debatidas na literatura internacional. Além disso, a utilização de um instrumento na investigação do estilo de vida, traduzido e validado para adultos brasileiros, pode subsidiar e servir de comparação para futuras investigações com a população universitária em geral.

Ademais, pode-se concluir que a prevalência de sobrepeso encontrada no presente estudo segue a tendência de outros países e serve de alerta, principalmente, por se tratar de pessoas jovens. Os grupos com maiores riscos para apresentar excesso de peso foram universitários do sexo masculino, estudantes com 20 anos de idade ou mais e casados. Em relação aos indicadores do estilo de vida, os sujeitos que reportaram estar no intervalo de mais de $2 \mathrm{~kg}$ do peso considerado saudável e os que ingerem bebidas com cafeína 
mais de duas vezes por dia tiveram mais chance de apresentar sobrepeso.

Recomenda-se que as instituições de ensino superior do Brasil realizem pesquisas prospectivas com a população universitária para identificar como se comporta a prevalência de sobrepe- so durante os anos acadêmicos. Além disso, sugere-se que a instituição investigada realize orientações e disponibilize programas de promoção da saúde no decorrer de todo período acadêmico, objetivando a redução do sobrepeso entre universitários.

\section{Colaboradores}

DAS Silva, TMB Quadros, AP Gordia e EL Petroski participaram igualmente de todas as etapas de elaboração do artigo.

\section{Referências}

1. Anderson P. Reducing overweight and obesity: closing the gap between primary care and public health. Fam Pract 2008; 25(Supl. 1):10-16.

2. Razak F, Anand SS, Shannon H, Vuksan V, Davis B, Jacobs R, Teo KK, McQueen M, Yusuf S. Defining obesity cut points in a multiethnic population. Circulation 2007; 115(16):2111-2118.

3. Organização Mundial da Saúde (OMS). Obesity and overweight. Fact sheet no 311. 2006. [acessado $2009 \mathrm{fev}$ 19]. Disponível em: http://www.who.int/mediacentre /factsheets/fs311/en/

4. Janghorbani M, Amini M, Rezvanian H, Gouya MM, Delavari A, Alikhani S, Mahdavi A. Association of body mass index and abdominal obesity with marital status in adults. Arch Iran Med 2008; 11(3):274-281.

5. American College Health Association. American College Health Association National College Health Assessment Spring 2006 Reference Group data report (abridged). J Am Coll Health 2007; 55:195-206.

6. Serlachius A, Hamer M, Wardle J. Stress and weight change in university students in the United Kingdom. Physiol Behav 2007;92(4):548-553.

7. Jung ME, Bray SR, Martin Ginis KA. Behavior change and the freshman 15: tracking physical activity and dietary patterns in 1st-year university women. $J \mathrm{Am}$ Coll Health 2008; 56(5):523-530.

8. Economos CD, Hildebrandt ML, Hyatt RR. College freshman stress and weight change: differences by gender. Am J Health Behav 2008; 32(1):16-25.

9. Delinsky SS, Wilson GT. Weight gain, dietary restraint, and disordered eating in the freshman year of college. Eat Behav 2008(1);9:82-90.

10. Racette SB, Deusinger SS, Strube MJ, Highstein GR, Deusinger RH. Changes in weight and health behaviors from freshman through senior year of college. J Nutr Educ Behav 2008; 40(1):39-42.

11. Brunt AR, Rhee YS. Obesity and lifestyle in U.S. college students related to living arrangemeents. Appetite 2008;51(3):615-621.

12. Blair SN, Horton E, Leon AS, Lee I-MIN, Drinkwater BL, Dishman RK, Mackey M, Kienholz ML. Physical activity, nutrition, and chronic disease. Med Sci Sports Exerc 1996; 28(3):335-349.

13. Canadian Society for Exercise Physiology (CSEF). The Canadian Physical Activity, Fitness and Lifestyle Appraisal: CSEP's guide to health active living. 2nd ed. Ottawa: CSEF; 2003. 
14. Rozmus CL, Evans R, Wysochansky M, Mixon D. An analysis of health promotion and risk behaviors of freshman college students in a rural southern setting. J Pediatr Nurs 2005; 20(1):25-33.

15. Luiz RR, Magnanini MMF. The logic of sample size determination in epidemiological research. Reports on Collective Health 2000; 8(2):9-28.

16. Coqueiro RS, Borges LJ, Araújo, VC, Pelegrini A, Barbosa, AR. Medidas auto-referidas são válidas para avaliação do estado nutricional na população brasileira? Rev Bras Cineantropom Desempenho Hum 2009; 11(1):113-119.

17. World Health Organization (WHO). Obesity: preventing and managing the global epidemic. Report of a WHO Consultation on Obesity (WHO Technical Report Series 894). Geneva: WHO; 2000.

18. Rodriguez-Añez CR, Reis RS, Petroski EL. Brazilian version of a lifestyle questionnaire: translation and validation for young adults. Arq Bras Cardiol 2008; 91(2):92-98.

19. Kamien M, Power R. Lifestyle and health habits of fourth year medical students at the University of Western Australia. Aust Fam Physician 1996; Supl. 1:S26-29.

20. Al-Kandari F, Vidal VL, Thomas D. Health-promoting lifestyle and body mass index among College of Nursing students in Kuwait: a correlational study. Nurs Health Sci 2008; 10(1):43-50.

21. Holm-Denoma JM, Joiner TE, Vohs KD, Heatherton TF. The "freshman fifteen" (the "freshman five" actually): predictors and possible explanations. Health Psychol 2008; 27(Supl. 1):S3-9.

22. Hajian-Tilaki KO, Heidari B. Prevalence of obesity, central obesity and the associated factors in urban population aged 20-70 years, in the north of Iran: a population-based study and regression approach. Obes Rev 2007; 8(1):3-10.

23. Sibai AM, Hwalla N, Adra N, Rahal B. Prevalence of and covariates of obesity in Labanon: finding from the first epidemiological study. Obes Res 2003; 11(11):1353-1361.

24. Lipowicz A, Gronkiewicz S, Malina RM. Body mass index, overweight and obesity in married and never married men and women in Poland. Am J Hum Biol 2002; 14(4):468-475.
25. Deshmukh-Taskar P, Nicklas TA, Yang SJ, Berenson GS. Does food group consumption vary by differences in socioeconomic, demographic, and lifestyle factors in young adults? The Bogalusa Heart Study. J Am Diet Assoc 2007; 107(2):223-234.

26. Roy JL, Hunter GR, Blaudeau TE. Percent body fat is related to body-shape perception and dissatisfaction in students attending an all women's college. Percept Mot Skills 2006; 103(3):677-684.

27. Diepvens K, Westerterp KR, Westerterp-Plantenga MS. Obesity and thermogenesis related to the consumption of caffeine, ephedrine, capsaicin, and green tea. Am J Physiol Regul Integr Comp Physiol 2007; 292(1):R77-85.

28. Rush E, Schulz S, Obolonkin V, Simmons D, Plank L. Are energy drinks contributing to the obesity epidemic? Asia Pac J Clin Nutr 2006; 15(2):242-244.

29. Dhingra R, Sullivan L, Jacques PF, Wang TJ, Fox CS, Meigs JB, D’Agostino RB, Gaziano JM, Vasan RS. Soft drink consumption and risk of developing cardiometabolic risk factors and the metabolic syndrome in middle-aged adults in the community. Circulation 2007; 116(5):480-488.

30. Seicean A, Redline S, Seicean S, Kirchner HL, Gao Y, Sekine M, Zhu X, Storfer-Isser A. Association between short sleeping hours and overweight in adolescents: results from a US Suburban High School survey. Sleep Breath 2007; 11(4):285-293.

31. Shaw K, Gennat H, Rourke PO, Del Mar C. Exercise for overweight or obesity. Cochrane Database Syst Rev 2006; 18(4):CD003817.

32. Brunner EJ, Rees K, Ward K, Burke M, Thorogood M. Dietary advice for reducing cardiovascular risk. Cochrane Database Syst Rev 2007; 17(4):CD002128

Artigo apresentado em 02/04/2009

Aprovado em 10/09/2009

Versão final apresentada em 25/09/2009 
